\title{
THEORETICAL DESCRIPTION OF A \\ REPARAMATRIZATION OF DISCRETE TWO \\ PARAMETER POISSON LINDLEY \\ DISTRIBUTION FOR MODELING WAITING AND \\ SURVIVAL TIMES DATA \\ Tanka Raj Adhikari*
}

ABSTRACT

In this research paper, the theoretical description of a reparamatrization of a discrete two-parameter Poisson Lindley Distribution, of which Sankaran's (1970) one parameter Poisson Lindley Distribution is a special case, is derived by compounding a Poisson Distribution with two parameters Lindley Distribution for modeling waiting and survival times data of Shanker et al. (2012).The first four moments of this distribution have derived. Estimation of the parameters by using method of moments and maximum likely hood method has been discussed.

Key Words: Compounding, reparamatrization, moments, estimation of parameters, maximum likely hood, probability generating function, moment generating function, two-parameter Lindley distribution.

\section{INTRODUCTION}

Lindley (1958) introduced a one parameter Lindley distribution, given by its probability density function

$f(x ; \theta)=\frac{\theta^{2}}{\theta+1}(1+x) e^{-\theta x}, x>0, \theta>0(1.1)$

Similarly one parameter Poisson Lindley distribution (PLD) given by its probability mass function as

$f(x ; \theta)=\frac{\theta^{2}(x+\theta+2)}{(\theta+1)^{x+3}}, x=0,1,2, \ldots ; \theta>0(1.2)$

This distribution has been introduced by Sankaran (1970) to model count data.

The distribution arises from a Poisson distribution when its parameter follows a Lindley distribution (1.1).

There paramatrization one parameter PLD is given by probability mass function as

$f(x ; \theta)=(1+2 \theta+\theta x) \frac{\theta^{x}}{(1+\theta)^{x+3}}, x=0,1,2, \ldots ; \theta>0(1.3)$

Recently, Shanker et al. (2012) obtained a two parameter Lindley distribution given by the probability density function

Dr. Adhikari is Reader at Department of Statistics, P. N. Campus, Pokhara, Nepal 
$f(x ; \alpha, \theta)=\frac{\theta^{2}}{\theta+\alpha}(1+\alpha x) e^{-\theta x}, x>0, \theta>0, \alpha>0(1.4)$

For $\alpha=1$, the distribution reduces to the one parameter Poisson Lindley distribution. This distribution has been found to be a better model then one parameter PLD for analyzing waiting and survival times and grouped mortality data.

Suppose that the parameter $\lambda$ of a Poisson distribution follows the two parameter LD (1.4). Then the two parameter Lindley mixture of Poisson distribution becomes

$f(x ; \lambda, \theta)=\int_{0}^{\infty} \frac{e^{-\lambda} \lambda^{x}}{\Gamma(1+x)} \cdot \frac{\theta^{2}}{\theta+\alpha}(1+\alpha \lambda) e^{-\theta \lambda} d \lambda, x>0, \alpha>0, \lambda>0, \theta>0(1.5)_{\mathrm{a}}$

$=\frac{\theta^{2}}{(\theta+1)^{x+2}}\left[1+\frac{\alpha x+1}{\theta+\alpha}\right], x=0,1,2, \ldots ; \theta>0, \alpha>-\theta(1.6)_{\mathrm{a}}$

Similarly, the reparamatrization of two parameter Lindley mixture of Poisson distribution becomes

$$
\begin{aligned}
& f(x ; \lambda, \theta)=\int_{0}^{\infty} \frac{e^{-\lambda} \lambda^{x}}{\Gamma(1+x)} \cdot \frac{1}{\theta(1+\alpha \theta)}(1+\alpha \lambda) e^{-\theta / \lambda} d \lambda, x>0, \alpha>0, \lambda>0, \theta>0(1.5)_{\mathrm{b}} \\
& =\frac{\theta^{x}}{(\theta+1)^{x+2}}\left[1+\frac{\theta(\alpha x+1)}{1+\alpha \theta}\right], x=0,1,2, \ldots ; \theta>0, \alpha>-\theta(1.6)_{\mathrm{b}}
\end{aligned}
$$

It can be seen that for $\alpha=1$, this distribution reduces to the reparatrization one parameter PLD (1.3). for $\alpha=0$, it reduces to the geometric distribution,

$f\left(x ; \lambda, \frac{1}{\theta}\right)=\frac{\theta^{x}}{(\theta+1)^{x+1}}$, with parameter $p=\frac{\theta}{\theta+1}$.

\section{MOMENTS}

The rth moment about the origin of the reparamatrization two parameter PLD $(1.6)_{\mathrm{b}}$ can be obtained as

$\mu_{r}^{\prime}=E\left[x^{r} / \lambda\right](2.1)$

From the relation $(1.5)_{\mathrm{b}}$ we get,

$\mu_{r}^{\prime}=\int_{0}^{\infty}\left[\sum_{x=0}^{\infty} x^{r} \frac{e^{-\lambda} \lambda^{x}}{\Gamma(1+x)}\right] \cdot \frac{1}{\theta(1+\alpha \theta)}(1+\alpha \lambda) e^{-\lambda / \theta} d \lambda, x>0, \alpha>0, \lambda>0, \theta>0(2.2)$

Obviously the expression under bracket is the rth moment about origin of the Poisson distribution. Taking $\mathrm{r}=1$, in (2.2) and using the mean of the Poisson distribution, the mean of the reparamatrization discrete two parameter PLD is obtained as

$\mu_{1}^{\prime}=\frac{1}{\theta(1+\alpha \theta)} \int_{0}^{\infty} \lambda(1+\alpha \lambda) e^{-\lambda / \theta} d \lambda$

$=\frac{\theta(1+2 \alpha \theta)}{\theta(1+\alpha \theta)}$ 
Taking $r=2,3,4$ in (2.2) we get,

$\mu_{2}^{\prime}=\frac{1}{\theta(1+\alpha \theta)} \int_{0}^{\infty}\left(\lambda^{2}+\lambda\right)(1+\alpha \lambda) e^{-\lambda / \theta} d \lambda$

$\mu_{3}^{\prime}=\frac{\theta(1+2 \alpha \theta)}{\theta(1+\alpha \theta)}+\frac{6 \theta^{2}(1+3 \alpha \theta)}{1+\alpha \theta}+\frac{6 \theta^{3}(1+4 \alpha \theta)}{1+\alpha \theta}(2.5)$

$$
=\frac{\theta(1+2 \alpha \theta)}{1+\alpha \theta}+\frac{2 \theta^{2}(1+3 \alpha \theta)}{1+\alpha \theta}(2.4)
$$

and

$\mu_{4}^{\prime}=\frac{\theta(1+2 \alpha \theta)}{\theta(1+\alpha \theta)}+\frac{14 \theta^{2}(1+3 \alpha \theta)}{1+\alpha \theta}+\frac{36 \theta^{3}(1+4 \alpha \theta)}{1+\alpha \theta}+\frac{24 \theta^{4}(1+5 \alpha \theta)}{1+\alpha \theta}(2.6)$

It can be seen that at $\alpha=1$, the above moments reduce to the respective moments of the reparamatrization one-parameter PLD.

Probability Generating Function (PGF)

The probability generating function of the discrete two parameter PLD is given by;

$$
\begin{aligned}
P_{x}(t)=E\left(t^{x}\right)= & \frac{\theta^{2}}{(\theta+1)^{2}} \sum_{x=0}\left(\frac{\mathrm{t}}{\theta+1}\right)^{\mathrm{x}} \\
& +\frac{\theta^{2}}{(\theta+1)^{2}(\theta+\alpha)} \cdot \sum_{\mathrm{x}=\mathrm{o}}(\alpha \mathrm{x}+1)\left(\frac{\mathrm{t}}{\theta+1}\right)^{\mathrm{x}} \\
& =\frac{\theta^{2}(\theta+1-\mathrm{t})+\alpha \theta^{2}}{(\theta+1-\mathrm{t})^{2}(\theta+\alpha)}(2.7)_{\mathrm{a}}
\end{aligned}
$$

Its reparamatrization PGF is given by;

$$
\begin{aligned}
& P_{x}(t)=E\left(t^{x}\right)=\frac{\theta^{2}}{(\theta+1)^{2}} \sum_{\mathrm{x}=0}\left(\frac{\mathrm{t}}{\theta+1}\right)^{\mathrm{x}} \\
& +\frac{\theta^{2}}{(\theta+1)^{2}(\theta+\alpha)} \cdot \sum_{x=0}(\alpha x+1)\left(\frac{t}{\theta+1}\right)^{x} \\
& =\frac{1+\theta-\mathrm{t} \theta+\alpha \theta}{(\theta+1-\mathrm{t} \theta)^{2}(1+\alpha \theta)}(2.7)_{\mathrm{b}}
\end{aligned}
$$

\section{MOMENT GENERATING FUNCTION (MGF)}

The moment generating function of the discrete two parameter PLD is given by

$\mathrm{M}_{\mathrm{x}}(\mathrm{t})=\mathrm{E}\left(\mathrm{e}^{\mathrm{tx}}\right)=\frac{\theta^{2}\left(\theta+1-\mathrm{e}^{\mathrm{t}}\right)+\alpha \theta^{2}}{\left(\theta+1-\mathrm{e}^{\mathrm{t}}\right)^{2}(\theta+\alpha)}(2.8)_{\mathrm{a}}$ and its reparamatrization MGF is given by; 
$\mathrm{M}_{\mathrm{X}}(\mathrm{t})=\frac{1+\theta-\theta \mathrm{e}^{\mathrm{t}}+\alpha \theta}{\left(\theta+1-\theta \mathrm{e}^{\mathrm{t}}\right)^{2}(1+\alpha \theta)}(2.8)_{\mathrm{b}}$

\section{ESTIMATION OF PARAMETER}

In this section we derive estimators for the two parameter $\alpha$ and $1 / \theta$ we use two methods

\section{Estimation BASED ON THE METHOD OF MOMENTS:}

By using the relation (2.3) and (2.4) we get;

$\frac{\mu_{2}^{\prime}-\mu_{1}^{\prime}}{\mu_{1}^{\prime 2}}=\frac{2(1+3 \alpha \theta)(1+\alpha \theta)}{(1+2 \alpha \theta)^{2}}=\mathrm{k}$ (say)

Setting,

$\frac{1}{\theta}=\mathrm{b} \alpha \quad$ or $\frac{1}{\mathrm{~b}}=\alpha \theta$ in (3.1) we get;

$\frac{\mu_{2}^{\prime}-\mu_{1}^{\prime}}{\mu_{1}^{\prime 2}}=\frac{2(b+3)(b+1)}{(b+2)^{2}}=m$ (say)

$$
\begin{aligned}
& \text { Or, } 2 b^{2}+8 b+6=m b^{2}+4 m b+4 m \\
& \text { Or, }(2-m) b^{2}+(8-4 m) b+(6-4 m)=0
\end{aligned}
$$

Which is a quadratic equation in $b$.

Replacing the first two population moments by the respective sample moments in (3.1) an estimate $\mathrm{k}$ of $\mathrm{m}$ can be obtained. Using $\mathrm{m}$ in (3.2), an estimate $\hat{b}$ of $b$, can be obtained. It can be seen that estimates of $b$ can be obtained from (4.2) only when $\mathrm{m}<2$.

Again, substituting $\frac{1}{\theta}=b \alpha$ or, $\frac{1}{b}=\alpha \theta$ in the expression for mean (2.3) we get,

$\frac{\frac{1}{b \alpha}(1+2 b)}{1+b}=\frac{(1+2 b)}{\alpha b(b+1)}=\bar{X}$, and thus an estimator of $\alpha$ and $\theta$ are obtained as:

$\hat{\alpha}=\left(\frac{1+2 b}{b(1+b)}\right) \frac{1}{\bar{X}} \hat{\beta}=\frac{1}{b \widehat{\alpha}}=\left(\frac{b+1}{1+2 b}\right) \bar{X}$.

\section{Estimation USING THE MAXIMUM LiKELIHOOd METHOD:}

Let, $\mathrm{x}_{1}, \mathrm{x}_{2}, \ldots, \mathrm{x}_{\mathrm{n}}$ be a random sample of size $\mathrm{n}$ from the reparamatrization of the discrete two-parameters PLD (1.6) and let $f_{x}$ be the observed frequency in the sample corresponding to $\mathrm{X}=\mathrm{x}(\mathrm{x}=1,2, \ldots, \mathrm{k})$ such that $\sum_{1}^{k} f_{x}=n$, where $\mathrm{k}$ is the largest observed value having non-zero frequency. The likelihood function, $\mathrm{L}$ of the reparamatrization of the discrete two-parameter PLD (1.6) ${ }_{b}$ is given by

$$
L=\frac{\theta^{\sum_{x=1}^{k} x}}{(1+\theta)^{\sum_{x=1}^{k}(x+2) f_{x}}} \prod_{x=1}^{k}[(\theta+1)+\alpha \theta(x+1)]^{f_{x}}
$$

and the log likelihood function becomes 
$\log L=n \bar{x} \log \theta-\sum_{x=1}^{k} f_{x}(\mathrm{x}+2) \log (\theta+1)+\sum_{x=1}^{k} f_{x} \log [(\theta+$ 1) $+\alpha \theta(x+1)]$

The resultant two log likelihood equations are thus obtained as:

$\frac{\partial \log L}{\partial \theta}=\frac{n \bar{x}}{\theta}+\sum_{x=1}^{k} \frac{\alpha(x+1) f_{x}}{[(\theta+1)+\alpha \theta(x+1)]}=0$

and,

$$
\frac{\partial \log L}{\partial \alpha}=\sum_{x=1}^{k} \frac{\theta(x+1) f_{x}}{[(\theta+1)+\alpha \theta(x+1)]}=0
$$

The two equations (3.6) and (3.7) given above do not seem to be solved directly. However, the Fisher's scoring method can be applied to solve these equations. For this, we have:

$$
\begin{aligned}
& \frac{\partial^{2} \log L}{\partial \theta^{2}}=-\frac{n \bar{x}}{\theta^{2}}-\sum_{x=1}^{k} \frac{\alpha(x+1)\{1+\alpha(x+1)\} f_{x}}{[(\theta+1)+\alpha \theta(x+1)]^{2}}(3.8) \\
& \frac{\partial^{2} \log L}{\partial \theta \partial \alpha}=\sum_{x=1}^{k} \frac{(x+1) f_{x}}{[(\theta+1)+\alpha \theta(x+1)]^{2}}(3.9) \\
& \frac{\partial^{2} \log L}{\partial \alpha^{2}}=-\sum_{x=1}^{k} \frac{\{\theta(x+1)\} f_{x}}{[(\theta+1)+\alpha \theta(x+1)]^{2}}(3.10)
\end{aligned}
$$

The equations can be solved by an iteration procedure to obtain maximum likely hood estimators of $1 / \hat{\theta}$ and $\hat{\alpha}$ starting with initial values $\theta_{0}$ and $\alpha_{0}$ of $\theta$ and $\alpha$, respectively.

$$
\left[\begin{array}{ll}
\frac{\partial^{2} \log L}{\partial \theta^{2}} & \frac{\partial^{2} \log L}{\partial \alpha \partial \theta} \\
\frac{\partial^{2} \log L}{\partial \alpha \partial \theta} & \frac{\partial^{2} \log L}{\partial \alpha^{2}}
\end{array}\right]_{\substack{\hat{\theta}=\theta_{0} \\
\hat{\alpha}=\alpha_{0}}}\left[\begin{array}{c}
\hat{\theta}-\theta_{0} \\
\hat{\alpha}-\alpha_{0}
\end{array}\right]=\left[\begin{array}{c}
\frac{\partial \log L}{\partial \theta} \\
\frac{\partial \log L}{\partial \alpha}
\end{array}\right]_{\substack{\hat{\theta}=\theta_{0} \\
\hat{\alpha}=\alpha_{0}}}
$$

\section{CONCLUSION}

In this paper, the writer proposed a reparamatrization of a twoparameter PLD of which one parameter is a particular case, for modeling waiting and survival times data. Several properties of this PLD such as moments, probability generating function, moment generating function and estimation of parameters by using method of moments and the method of maximum likelihood have been discussed. This distribution can be fitted by using chi-square test for appropriate waiting and survival times data.

\section{WORKS CITED}

Beall, G. (1940). "The Fit and Significance of Contagious when Applied to Observations on Larval Insects." Ecology.21, 460-474.

Borah, M. (1984). "The Gegenbauer Distribution Revisited: Some Recurrence Relation for Moments, Cumulants, etc., Estimation of Parameters and its Goodness of Fit." Journal of Indian Society of Agricultural Statistics.36, $72-78$. 
Bjerkedal, T. (1960). "Acquisition of Resistance in Guinea Pigs Infected with Different Doses of Virulent Tubercle Ba-cilli." American Journal of Epidemiology. Vol. 72, No. 1, pp. 130-148.

Deniz, E.G. and Ojeda, E.C. (2011). "The Discrete Lindley DistributionProperties and Applications."Journal of Statistical Computation and Simulation. Vol. 81, No. 11, pp. 1405-1416.

Ghitany, M.E. and Al-Mutairi, D.K. (2009). "Estimation Methods for the Discrete Poisson-Lindley Distribution." Journal of Statistical Computation and Simulation. 79(1), 1-9.

Ghitany, M.E., Alqallaf, F., Al-Mutairi, D.K. and Hussain, H.A. (...). "A two Parameter Weighted Lindley Distribution and its Applications to Survival Data. "Mathematics and Computers in Simulation. Vol. 81, No. 6, pp. 1190-1201.

Ghitany, M.E., Atieh, B. and Nadarajah, S. (2008). "Lindley Distribution and its Applications." Mathematics and Computers in Simulation. Vol. 78, No. 4, pp. 493-506.

Kemp, C.D. and Kemp, A.W. (1965). "Some Properties of the Hermite Distribution. "Biometrika.52, 381-394.

Lindley, D.V. (1958). "Fiducial Distribution and Bayes Theorem." Journal of Royal Statistical Society. Series B, 20, No.1, 102-107.

Shankaran, M. (1970). "The discrete Poisson-Lindley Distribution." Biometric. Vol. 26, No. 1, pp. 145-149.

Shanker, R., Sharma, S. and Shanker, R. (2012) ${ }^{1}$. A Two-parameter Lindley Distribution for Modeling Waiting and Survival Times Data. (Accepted for Publication in Applied Mathematics).

--- $(2012)^{2}$. A Discrete Two-parameter Poisson Lindley Distribution: JESA, Vol. XXI, pp. 15-22.

--- (2013). ATtwo-parameter Lindley Distribution for Modeling Waiting and Survival Times Data; doi: 10.4236/am. 2013, 42056 Published Online February 2013 (http://www.scirp.org/ journal/am) Applied Mathematics, 2013, 4 363-368. 